\title{
Application of the Total Least Square ESPRIT Method to Estimation of Angular Coordinates of Moving Objects
}

\author{
Wojciech Rosloniec \\ Faculty of Electronics and Information Technology, Warsaw University of Technology, Nowowiejska 15/19, 00-665 Warsaw, Poland \\ Correspondence should be addressed to Wojciech Rosloniec, wrosloni@poczta.onet.pl \\ Received 3 September 2010; Accepted 22 December 2010 \\ Academic Editor: Tat Yeo \\ Copyright () 2010 Wojciech Rosloniec. This is an open access article distributed under the Creative Commons Attribution License, \\ which permits unrestricted use, distribution, and reproduction in any medium, provided the original work is properly cited.
}

The TLS ESPRIT method is investigated in application to estimation of angular coordinates (angles of arrival) of two moving objects at the presence of an external, relatively strong uncorrelated signal. As a radar antenna system, the 32-element uniform linear array (ULA) is used. Various computer simulations have been carried out in order to demonstrate good accuracy and high spatial resolution of the TLS ESPRIT method in the scenario outlined above. It is also shown that accuracy and angle resolution can be significantly increased by using the proposed preprocessing (beamforming). The most of simulation results, presented in a graphical form, have been compared to the corresponding equivalent results obtained by using the ESPRIT method and conventional amplitude monopulse method aided by the coherent Doppler filtration.

\section{Introduction}

One of the important problems of today's radar technology is the detection and estimation of angular coordinates of fast moving objects, the radar echoes of which are received on a background of strong uncorrelated interferences and signals reflected from other objects located nearby. It is obvious that in such situations the conventional monopulse methods are rather noneffective $[1,2]$. This noneffectiveness results from the essence of them, since they enable the correct estimation of angular coordinates merely in situations when just one sufficiently strong useful signal is being received in a specific moment. Unfortunately, in the real electromagnetic environment, besides the useful signal (reflected from a detected object), different spurious signals are also received by the radar antenna unit. For instance, these spurious signals may be reflected from the earth, weather precipitation, or other masking objects flying around. It follows from the literature that problems of this kind can be effectively solved by modern radar systems using the angular superresolution methods. Thus, in this paper, one of them, namely, the Total Least Square ESPRIT (TLS ESPRIT), has been used for this aim [3-6]. The corresponding theory is presented in Section 2. In the next, that is, Section 3, it is shown how this method can be effectively applied to solve different variants of the general problem formulated above. A novel component of the proposed approach is an algorithm of preprocessing the samples of signals received simultaneously by a linear uniform antenna array. This algorithm creates a new "virtual" linear array with smaller number of radiating/receiving elements. It is important that signal-to-noise ratio evaluated at each of its virtual elements is greater, thanks to integration of signals from consecutive radiating/receiving elements of the original array. Consequently, the angular coordinates of the detected object can be evaluated more accurately. The angular resolution of the applied method is also increased. A short discussion on the results of the corresponding computer simulations is presented in Sections 3, 4, 5, 6, and 7 .

\section{TLS ESPRIT Method}

The TLS ESPRIT method can be treated as an improved version of the ESPRIT method, the theoretical basis of which is described in many publications, for example, [5-7]. To recall the essence of the TLS ESPRIT method, let us assume that the signals arriving from different directions are received 
by two identical subarrays, $X$ and $Y$, mutually displaced by distance $\Delta$, Figure 1 .

The resultant signals received by the arrays are the sum of the $K$ signals $s_{k}(t), k=1, \ldots, K$ being received from specific directions determined by the angles $\theta_{k}$. The vectors of signals received by the first subarray $(X)$ and second subarray $(Y)$, as shown in Figure 1, are described by the following equations, respectively:

$$
\mathbf{x}(n)=\mathbf{A s}(n)+\mathbf{u}(n), \quad \mathbf{y}(n)=\mathbf{A} \boldsymbol{\Phi} \mathbf{s}(n)+\mathbf{v}(n),
$$

where

$$
\begin{aligned}
\mathbf{A} & =\left[\mathbf{a}\left(\theta_{1}\right), \mathbf{a}\left(\theta_{2}\right), \ldots, \mathbf{a}\left(\theta_{K}\right)\right] \\
& =\left[\begin{array}{cccc}
a_{1}\left(\theta_{1}\right) e^{-j \omega_{0} \tau_{1}\left(\theta_{1}\right)} & a_{1}\left(\theta_{2}\right) e^{-j \omega_{0} \tau_{1}\left(\theta_{2}\right)} & \ldots & a_{1}\left(\theta_{K}\right) e^{-j \omega_{0} \tau_{1}\left(\theta_{K}\right)} \\
a_{2}\left(\theta_{1}\right) e^{-j \omega_{0} \tau_{2}\left(\theta_{1}\right)} & a_{2}\left(\theta_{2}\right) e^{-j \omega_{0} \tau_{2}\left(\theta_{2}\right)} & \cdots & a_{2}\left(\theta_{K}\right) e^{-j \omega_{0} \tau_{2}\left(\theta_{K}\right)} \\
\vdots & \vdots & \ddots & \vdots \\
a_{N-1}\left(\theta_{1}\right) e^{-j \omega_{0} \tau_{N-1}\left(\theta_{1}\right)} & a_{N-1}\left(\theta_{2}\right) e^{-j \omega_{0} \tau_{N-1}\left(\theta_{2}\right)} & \cdots & a_{N-1}\left(\theta_{K}\right) e^{-j \omega_{0} \tau_{N-1}\left(\theta_{K}\right)}
\end{array}\right],
\end{aligned}
$$

where $\omega_{0}$ is the angular frequency of carrier wave of the signal received by the antenna array, $\lambda$ is the wavelength of the carrier wave of the signal received by the antenna array, $a_{i}\left(\theta_{k}\right)$ is the parameter representing the directional gain of the $i$ th radiating element, and $\tau_{i}\left(\theta_{k}\right)$ is the delay of the signal reaching the $i$ th radiating element,

$$
\Phi=\left[\begin{array}{cccc}
e^{-j 2 \pi \Delta / \lambda \sin \left(\theta_{1}\right)} & 0 & \cdots & 0 \\
0 & e^{-j 2 \pi \Delta / \lambda \sin \left(\theta_{2}\right)} & \cdots & 0 \\
\vdots & \vdots & \ddots & \vdots \\
0 & 0 & \cdots & e^{-j 2 \pi \Delta / \lambda \sin \left(\theta_{K}\right)}
\end{array}\right]
$$

diagonal matrix of the scaling coefficients,

having $\mathbf{s}(n)$ as the vector of samples received by the antenna array, $\mathbf{s}(n)=\left[s_{1}(n), s_{2}(n), \ldots, s_{K}(n)\right]^{T}, \mathbf{u}(n)$ as the vector of noise samples in the radiating elements of the first subarray, and $\mathbf{v}(n)$ as the vector of noise samples in the radiating elements of the second subarray.

The vector of samples $\mathbf{z}(n)$, which is composed of samples of signals received from both subarrays, is defined as follows:

$$
\mathbf{z}(n)=\left[\begin{array}{l}
\mathbf{x}(n) \\
\mathbf{y}(n)
\end{array}\right]=\overline{\mathbf{A}} \mathbf{s}(n)+\mathbf{n}(n),
$$

where

$$
\overline{\mathbf{A}}=\left[\begin{array}{c}
\mathbf{A} \\
\mathbf{A} \boldsymbol{\Phi}
\end{array}\right], \quad \mathbf{n}(n)=\left[\begin{array}{l}
\mathbf{u}(n) \\
\mathbf{v}(n)
\end{array}\right]
$$

For the vector $\mathbf{z}(n)$, the spatial correlation matrix is formulated

$$
\mathbf{R}_{Z}=\overline{\mathbf{A}} \mathbf{S} \overline{\mathbf{A}}^{H}+\sigma^{2} \boldsymbol{\Sigma},
$$

where $\mathbf{S}=E\left\{\mathbf{s}(n) \mathbf{s}(n)^{H}\right\}$ and $\boldsymbol{\Sigma} \equiv \mathbf{I}=\operatorname{diag} .\{1,1,1, \ldots, 1\}$ is a unitary matrix of the $(2 N-2)$-th degree. The generalized eigenvectors $\mathbf{v}_{i}, i=1, \ldots, 2 N-2$ of the matrix $\mathbf{R}_{Z}$ create a space, in which the directional vectors of signals $s_{k}(n)$, received by both $N-1$ element antenna arrays lie (Figure 1). All generalized eigenvectors $\mathbf{v}_{i}$ satisfy the equation

$$
\left(\mathbf{R}_{Z}-\lambda_{i} \boldsymbol{\Sigma}\right) \mathbf{v}_{i}=0
$$

where $\lambda_{i}$ denotes the $i$ th eigenvalue of matrix $\mathbf{R}_{Z}$. On the basis of eigenvectors corresponding to the $K$ biggest eigenvalues, the vector matrix of the signal space is created

$$
\mathbf{V}_{S}=\left[\mathbf{v}_{1}\left|\mathbf{v}_{2}\right| \cdots \mid \mathbf{v}_{K}\right]
$$

The vectors included in matrix (8) can be presented as a combination of the directional vectors:

$$
\mathbf{V}_{S}=\overline{\mathbf{A}} \mathbf{T}
$$

since the directional vectors of received signals (written into columns of matrix $\overline{\mathbf{A}}$ ) span over the same signal space. Like in the case of the matrix of directional vectors $\overline{\mathbf{A}}$, the eigenvector matrix $\mathbf{V}_{S}$ has the structure that allows us to determine the phase shift between the signals received by subarrays $X$ and $Y$, see Figure 1

$$
\mathbf{V}_{S}=\left[\begin{array}{l}
\mathbf{V}_{X} \\
\mathbf{V}_{Y}
\end{array}\right]=\left[\begin{array}{c}
\mathrm{AT} \\
\mathrm{A} \Phi \mathrm{T}
\end{array}\right] .
$$

That means that for the matrix $\mathbf{V}_{X Y}=\left[\mathbf{V}_{X} \mid \mathbf{V}_{Y}\right]$ exists a matrix

$$
\mathbf{F}=\left[\begin{array}{c}
\mathbf{F}_{X} \\
\mathbf{F}_{Y}
\end{array}\right], \quad \mathbf{F} \in C^{2 K \times K}
$$

fulfilling the following equation, $[6,8]$ :

$$
\mathbf{V}_{X Y} \mathbf{F}=\mathbf{V}_{X} \mathbf{F}_{X}+\mathbf{V}_{Y} \mathbf{F}_{Y}=\mathbf{A T F}_{X}+\mathbf{A} \boldsymbol{A} \mathbf{T F}_{Y}=\mathbf{0} .
$$

By using the matrix $\boldsymbol{\Psi}=-\mathbf{F}_{X} \mathbf{F}_{Y}^{-1}$, (12) can be written in the form $\mathrm{AT} \Psi=\mathrm{A} \Phi \mathrm{T}$, which results in

$$
\boldsymbol{\Psi}=\mathrm{T}^{-1} \boldsymbol{\Phi} \mathbf{T} .
$$




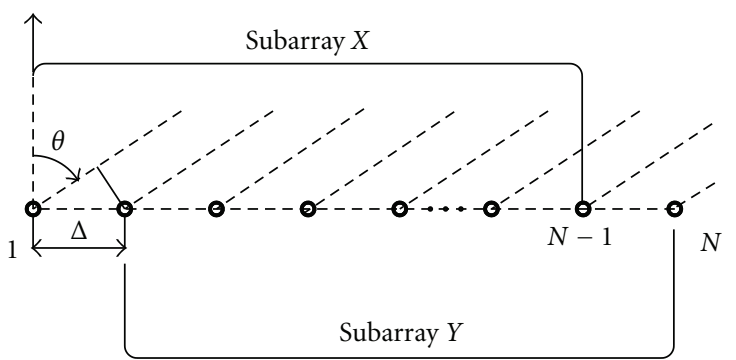

Figure 1: A uniform linear array antenna composed of $N$ sensors and their $(N-1)$-sensor subarrays $X$ and $Y$.

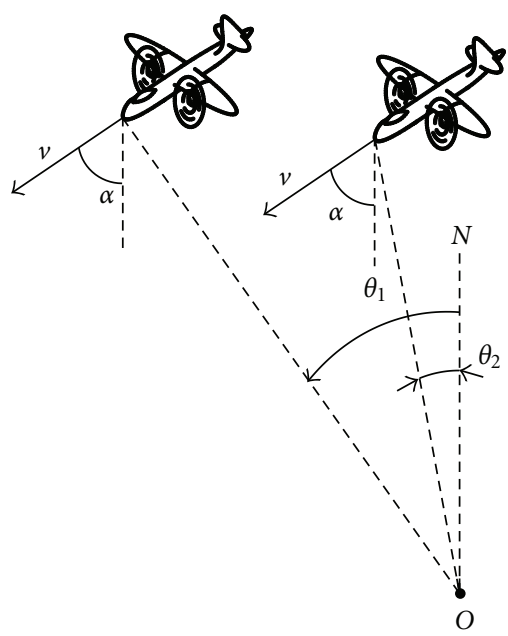

Figure 2: Polar coordinates (angles of arrival) of the planes (objects) being detected.

Here, it should be noticed that (13) has similar form as equation $\boldsymbol{\Psi}=\mathbf{V} \Lambda \mathbf{V}^{H}$, which defines eigenvalues and eigenvectors of matrix $\Psi$. Therefore, columns of matrix $\mathbf{T}^{-1}$ are equal to eigenvectors of matrix $\Psi$, and values of diagonal elements of matrix $\Phi$ are equal to related eigenvalues of matrix $\Psi$. When the values of the phase shifts included in the diagonal elements of $\Phi$ are known, one can determine the directions represented by the angles $\theta_{k}$, from which the signals $s_{k}(t)$ are received.

Unfortunately, the method described above requires a very accurate estimate of the correlation matrix $\mathbf{R}_{Z}$, for which the following equation should be satisfied:

$$
\mathbf{V}_{X} \Psi=\mathbf{V}_{Y}
$$

In practice, (14) is not satisfied precisely due to an inaccurate estimate of the correlation matrix $\mathbf{R}_{Z}$ determined on basis of limited number of measurements of the vector $\mathbf{z}(n)$. The estimate of the correlation (covariance) matrix is

$$
\widehat{\mathbf{R}}_{Z}=\frac{1}{N_{\text {ret }}} \sum_{k=1}^{N_{\text {ret }}} \mathbf{z}(g+k \cdot G) \mathbf{z}^{*}(g+k \cdot G),
$$

where $N_{\text {ret }}$ denotes the number of pulse returns (realizations) employed to estimate the values of the spatial correlation matrix, $g$ is the index of range gate, and $G$ is the number

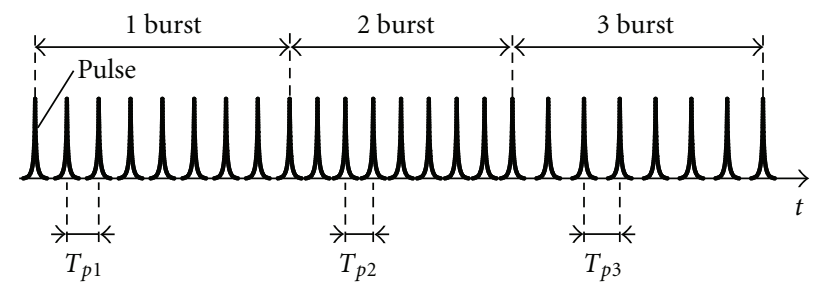

FIGURE 3: A structure of a radar signal used in the MTD processing.

of range gates obtained after sampling of the received pulse return. In such case, the generalized eigenvectors, obtained as the result of the decomposition of matrices $\mathbf{R}_{Z}$ and $\boldsymbol{\Sigma}$, are inaccurate. The sought matrix $\Psi$, for which the error of mapping the matrix $\mathbf{V}_{X}$ into matrix $\mathbf{V}_{Y}$ is the least, can be found using the criterion of the total least square error. According to this criterion, matrix $\mathbf{F}$ is evaluated, for which the value of square of the Euclidean (Frobenius) norm of the product of the matrices $\mathbf{V}_{X Y}$ and $\mathbf{F}$, that is

$$
P=\left\|\mathbf{V}_{X Y} \mathbf{F}\right\|_{F}^{2}=\left\|\mathbf{V}_{X} \mathbf{F}_{X}+\mathbf{V}_{Y} \mathbf{F}_{Y}\right\|_{F}^{2}
$$

is the least. Additionally, a limitation as described by the relationship below is imposed on the value of the matrix $\mathbf{F}$

$$
\mathbf{F}^{H} \mathbf{F}=\mathbf{I}
$$

The solution of the aforediscussed problem is the matrix $\mathbf{F}$ of dimension $2 K \times K$ composed of $K$ columns, which are equal to eigenvectors corresponding to $K$ least eigenvalues of the matrix product $\mathbf{V}_{X Y}^{H} \mathbf{V}_{X Y}$. The eigenvalues of the matrix $\Psi$ correspond to the sought values of the elements lying on the diagonal of the matrix $\Phi$. Knowing the values of diagonal elements of matrix $\boldsymbol{\Phi}$, values of angles $\theta_{k}$ can be found. To summarize, the TLS ESPRIT method can be implemented in the following way.

Step 1. Determine the estimate of the spatial correlation matrix $\mathbf{R}_{Z}$, based on the measurement of the signal vector $\mathbf{z}(n)$.

Step 2. Decompose the matrices $\mathbf{R}_{Z}$ and $\boldsymbol{\Sigma}$ with respect to their eigenvectors and eigenvalues, see (6) and (7), which satisfy the following condition:

$$
\hat{\mathbf{R}}_{Z} \mathbf{V}=\boldsymbol{\Sigma} \mathbf{V} \boldsymbol{\Lambda}
$$

where $\mathbf{V}$ is a matrix of eigenvectors and $\boldsymbol{\Lambda}$ is a diagonal matrix of eigenvalues.

Step 3. Determine the number of signal sources $K$.

Step 4. Out of the matrix $\mathbf{V}$, select vectors corresponding to $K$ biggest eigenvalues and create a matrix $\mathbf{V}_{s}$ of them. 


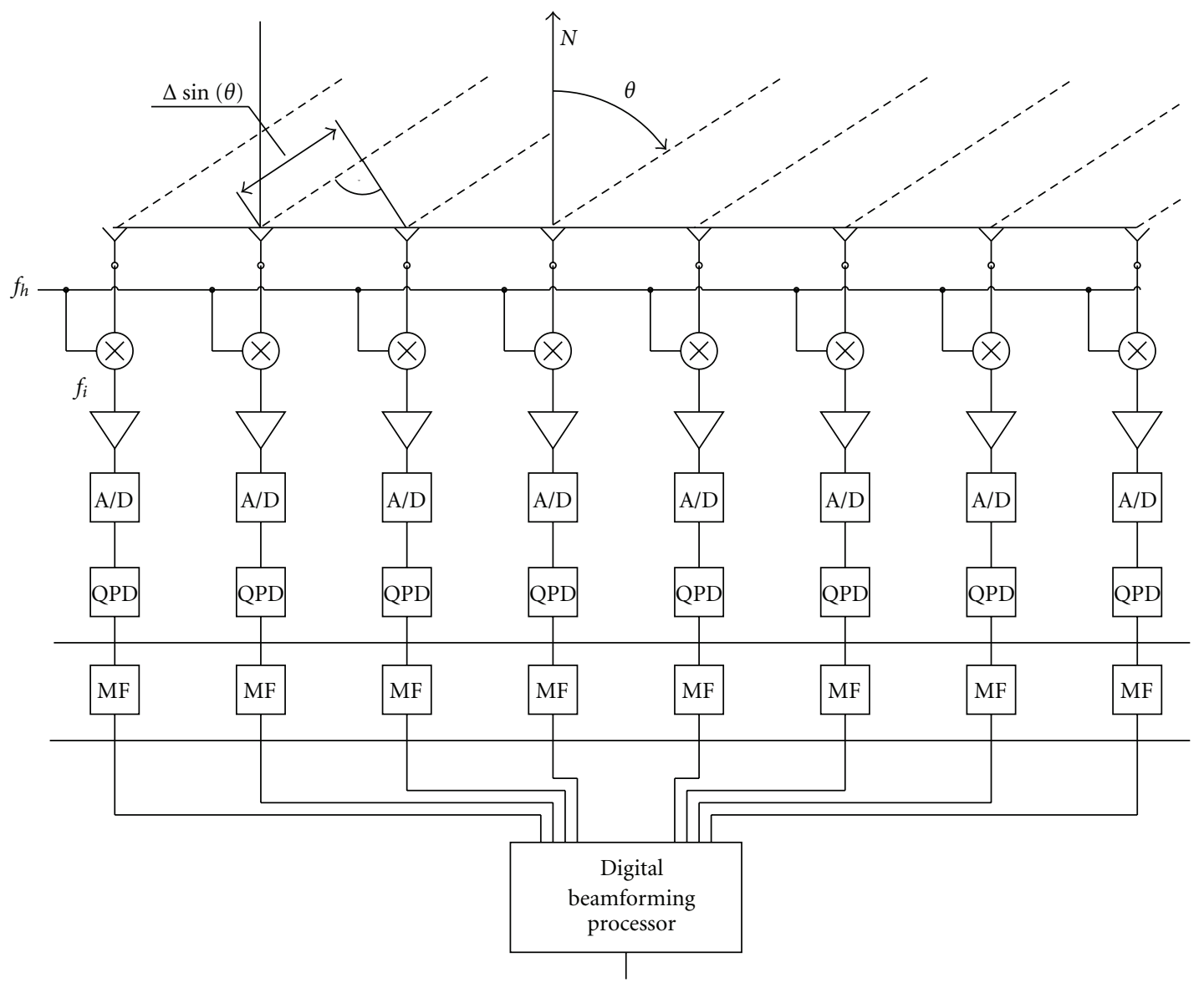

FIgURE 4: The functional scheme of an antenna unit including the digital beamformer. A/D: analog to digital converter, QPD: quadrature phase detector, MF: digital matching filter.

Step 5. Divide the matrix $\mathbf{V}_{s}$ into matrices $\mathbf{V}_{X}$ and $\mathbf{V}_{Y}$ and create a matrix $\mathbf{V}_{X Y}$ of them

$$
\mathbf{V}_{S}=\Sigma\left[\mathbf{v}_{1}, \mathbf{v}_{2}, \ldots, \mathbf{v}_{K}\right] \Longrightarrow\left[\begin{array}{c}
\mathbf{V}_{X} \\
\mathbf{V}_{Y}
\end{array}\right], \quad \mathbf{V}_{X Y}=\left[\mathbf{V}_{X} \mid \mathbf{V}_{Y}\right]
$$

Step 6. Decompose the matrix product $\mathbf{V}_{X Y}^{H} \mathbf{V}_{X Y}$ with respect to its eigenvectors and eigenvalues, which satisfy the equation $\mathbf{V}_{X Y}^{H} \mathbf{V}_{X Y}=\mathbf{E} \boldsymbol{\Lambda} \mathbf{E}$.

Step 7. Split the eigenvector matrix $\mathbf{E}$ into four submatrices of dimensions $K \times K$ each

$$
\mathbf{E}=\left[\begin{array}{ll}
\mathbf{E}_{11} & \mathbf{E}_{12} \\
\mathbf{E}_{21} & \mathbf{E}_{22}
\end{array}\right]
$$

Step 8. Based on the matrices $\mathbf{E}_{12}$ and $\mathbf{E}_{22}$, the columns of which correspond to the $K$ smallest eigenvalues, determine a matrix $\Psi$ by using the transformation $\Psi=-\mathbf{E}_{12} \mathbf{E}_{22}^{-1}$.

Step 9. Find the eigenvalues $\phi_{k}$ of matrix $\Psi$.
Step 10. Based on complex coefficients $\phi_{k}$, calculate the angles,

$$
\theta_{k}=\arcsin \left(\frac{c \cdot \operatorname{arctg}\left(\phi_{k}\right)}{\omega_{0} \Delta}\right), \quad k=1, \ldots, K,
$$

which determine the directions of arrival of signals $s_{k}(t)$, see Figure 1.

\section{Application of the TLS ESPRIT Method to Evaluate Angular Coordinates of Two Moving Objects}

In this section, the TLS ESPRIT method has been used to solve the problem of estimation of angular coordinates of two objects, moving with velocity $v=100 \mathrm{~m} / \mathrm{s}$, Figure 2 .

Let us assume that both objects bear at $\alpha=-45^{\circ}$ north and the azimuth angle of the first of them is $\theta_{1}=-14.1^{\circ}$. The azimuth angle $\theta_{2}$ of the second one is changing in the range of $-14.1^{\circ} \leq \theta_{2} \leq-10.1^{\circ}$, although without changing its bearing and velocity. Gradually the angular distance between these two objects, is changing in the range of $\Delta \theta=$ $0^{\circ} \div 4^{\circ}$. In order to estimate angular coordinates of these objects a radar system with electronically controlled antenna (nonrotating mechanically) has been used. This linear array 
is composed of $N=32$ radiating/receiving elements. The distance between two adjacent elements is equal to $\Delta=$ $0.7 \lambda$, where $\lambda$ is a length of the received electromagnetic wave. It is also assumed that the radar system performs signal processing using the MTD (moving target detection) algorithm, $[2,9]$. The processing is performed on the signal composed of 24 pulses grouped in three 8-pulse bursts which differ by their pulse repetition interval, Figure 3 . The number of pulses emitted by the radar in given direction is smaller than the number of elements of the array. In order to reduce an influence of inaccurate estimate of correlation matrix $\hat{\mathbf{R}}_{Z}$ on estimation of angular coordinates, the appropriate "diagonal loading" has been used (22)

$$
\hat{\mathbf{R}}_{Z \text { diag-load }}=\frac{1}{N_{\text {ret }}} \sum_{k=1}^{N_{\text {ret }}} \mathbf{z}(g+k \cdot G) \mathbf{z}^{*}(g+k \cdot G)+4 \sigma^{2} \cdot \mathbf{I},
$$

where $\sigma^{2}$ is the variance of noise in elements of the array and I is the identity matrix.

The signals received by the antenna array are used to form the sum and difference antenna patterns. Thus, the angular coordinates of objects being detected can be evaluated by means of amplitude monopulse method.

The samples $s(n)$ of echoes received by the sum beam $\boldsymbol{\Sigma}$ are subjected to coherent integration with the use of several sets of weight coefficients. The so-understood integration is called Doppler filtering, because it allows the discrimination of signals, which originate from objects moving with different radial velocities with respect to the radar. The signal obtained as a result of the so-understood integration is described by the general relationship:

$$
s_{\text {doppl }}(g)=\sum_{m=1}^{8} w_{m} s(g+m \cdot G),
$$

where $s_{\text {doppl }}(g)$ is the sample of signal in a given Doppler filter, $s(g)$ is the sample of the pulse return received by sum antenna beam, $g$ is the index of the range gate which determines the distance between the object and the radar, $G$ is the number of range gates, $m$ is the index of a pulse within the pulse sequence called "burst", and $w_{m}$ is appropriately chosen weight coefficient. After detection, that is, after distinguishing the range gates in which the level of detected signal exceeds a decision threshold, the radar system performs an estimation of angular coordinates. Naturally, it can be performed by using the conventional amplitude monopulse method. Moreover, the samples of signals appearing at sum and difference antenna outputs can be additionally filtered by the same Doppler filter. The more efficient solution uses the ESPRIT and TLS ESPRIT methods which both use the complex samples of the signal received by the 32-element antenna array; the samples form the vector $\mathbf{x}(n)$. The samples are obtained at outputs of matched filters MF, that is, before the beamforming network, see Figure 4 .

An attempt to evaluate angular coordinates of two closely spaced objects by means of the conventional amplitude monopulse method was met with failure. This failure

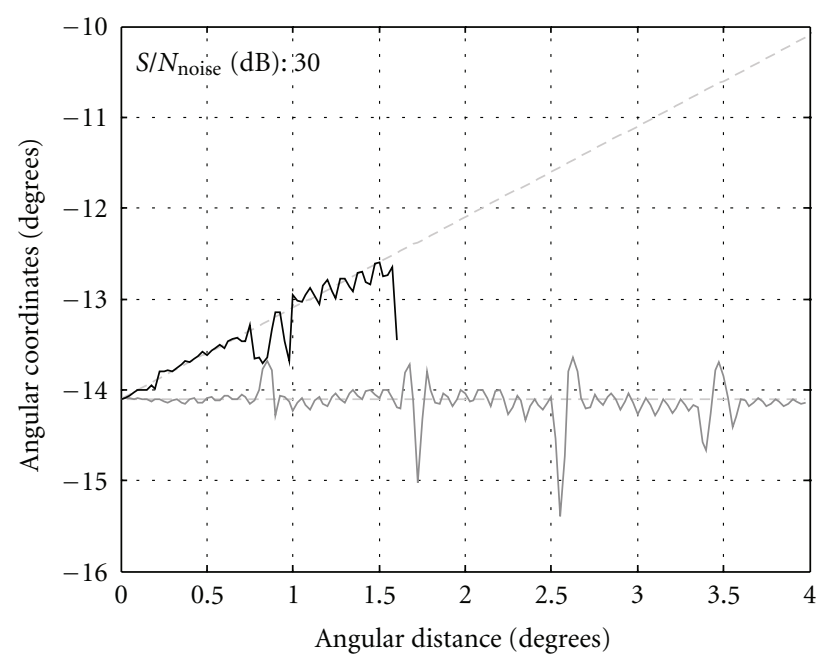

FIGURE 5: Histogram of polar coordinates evaluated by means of the amplitude monopulse method aided by the coherent Doppler filtration, $v=1000 \mathrm{~m} / \mathrm{s}, T_{p}=4 \mathrm{~ms}$, and $S / N_{\text {noise }}=30 \mathrm{~dB}$.

resulted from the fact that monopulse methods are not able to discriminate objects seen in the same antenna beam when these objects are located at a similar distance to the antenna. The angular resolution of angular coordinates of these objects is determined by $3 \mathrm{~dB}$ beam width of azimuth and elevation receive characteristics. Usually the second object, for instance, an aircraft causes errors of angular coordinates of the first one when it is seen within the same antenna beam. This effect can be partially suppressed using the additional Doppler filtering. It allows the attenuation of signals, which sustain another frequency shift due to Doppler effect. It is well known that a change of angular position of an object causes a change of the corresponding radial velocity and Doppler frequency shift related to it. Unfortunately, at the higher radial velocities, the normalized Doppler frequencies of echoes of these objects may be similar and cause errors of estimation of angular coordinates. This effect is illustrated in Figure 5. Thus, Doppler filtering can be used to increase signal-to-interference ratio when a radar receives an echo reflected from a single object together with interferences (weather clutter, ground clutter, etc.). However; it is not an appropriate approach to the resolution of two closely spaced objects. When these objects are moving with relatively small velocities (e.g., $v=100 \mathrm{~m} / \mathrm{s}$ ), their Doppler frequencies, normalized with respect to pulse repetition frequency (PRF) $f_{d \text {-norm }}=f_{d} \% F_{p}$, are different, but they may appear in $3 \mathrm{~dB}$ passband of the same Doppler filter. In the opposite situation, when two objects are moving with high speed (e.g., $v=1000 \mathrm{~m} / \mathrm{s}$ ) and PRF (e.g., $F_{p}=1 / T_{p}=250 \mathrm{~Hz}$ ) is small in comparison to their Doppler frequencies, the normalized Doppler frequencies of corresponding echoes may be similar or equal. The errors caused by this effect are illustrated in Figure 5.

For comparison, Figure 6 presents the diagrams of the estimates of the angular coordinates of two closely spaced objects found using the TLS ESPRIT method. 


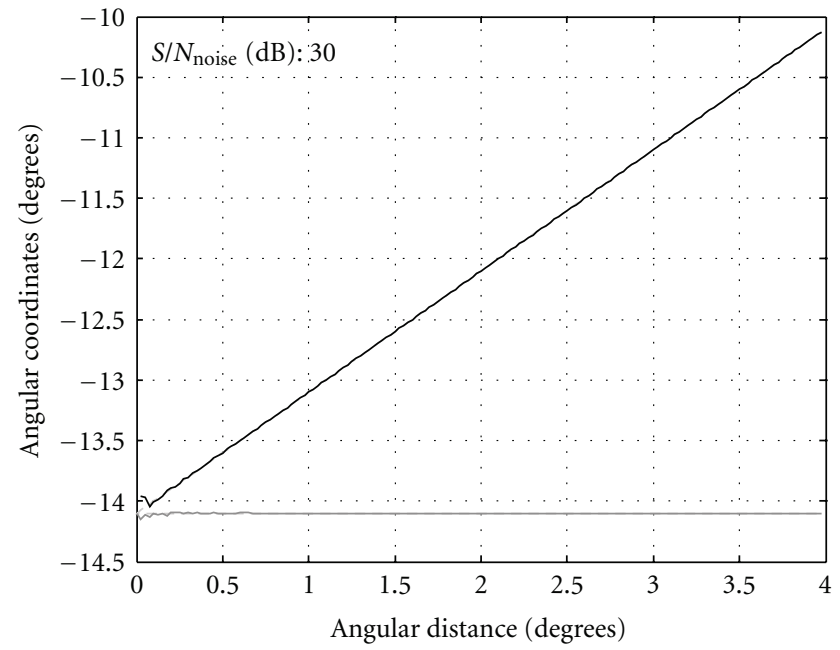

FIGURE 6: Histogram of polar coordinates evaluated by using the TLS ESPRIT method for the plane velocity $v=1000 \mathrm{~m} / \mathrm{s}, T_{p}=$ $4 \mathrm{~ms}$, and $S / N_{\text {noise }}=30 \mathrm{~dB}$.

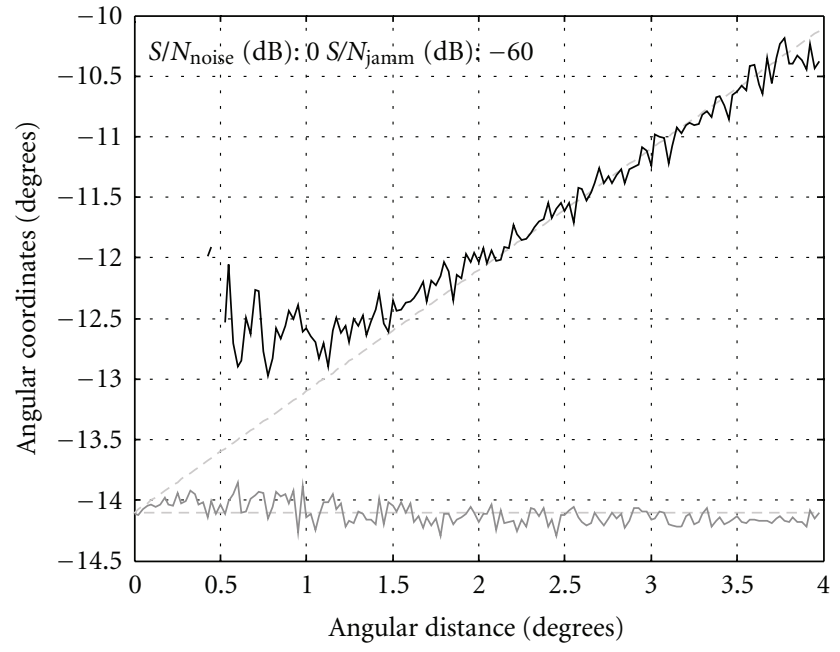

FIgURE 7: Histogram of polar coordinates evaluated by using the ESPRIT method at $S / N_{\text {noise }}=0 \mathrm{~dB}$ and $S / N_{\text {jamm }}=-60 \mathrm{~dB}$.

\section{The Preprocessing Preceding the TLS ESPRIT Method}

The results illustrated in Figure 6 have been obtained using the TLS ESPRIT method for signal samples recorded at outputs of the 24-element equivalent (virtual) antenna array. This "virtual" antenna array has been created in the manner described below, known as preprocessing. The samples of signals $x_{i}(n)$, where $1 \leq i \leq 32$, received by the real 32element antenna array, are used to compute the following samples:

$$
x_{k}^{(e)}(n)=\sum_{i=0}^{8} \frac{w_{i} x_{k+i}(n)}{\sqrt{9}}, \quad \text { for } 1 \leq k \leq 24 .
$$

Samples (24) correspond to particular elements of the 24element antenna array being formed. According to Figure 1,

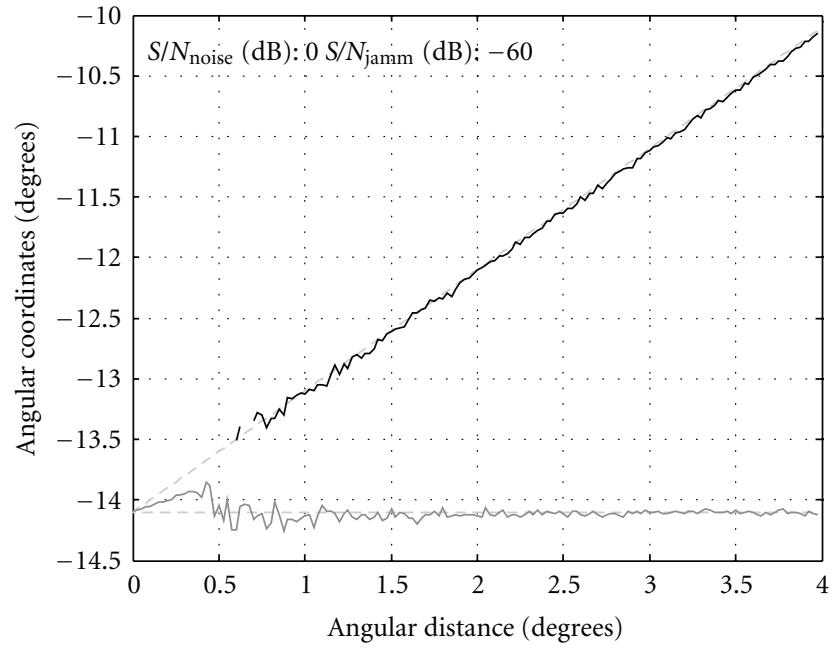

FIGURE 8: Histogram of polar coordinates evaluated by using the TLS ESPRIT method at $S / N_{\text {noise }}=0 \mathrm{~dB}$ and $S / N_{\text {jamm }}=-60 \mathrm{~dB}$.

TABLE 1: The angular resolution and mean-square error of angular coordinates evaluated by means of the TLS ESPRIT method.

\begin{tabular}{lccc}
\hline $\begin{array}{l}\text { Angular resolution, } \\
\text { degrees (estimation } \\
\left.\text { error }<0.23^{\circ}\right)\end{array}$ & $\begin{array}{c}\text { Maximum value of } \\
\text { estimation error, } \\
\text { degrees }\end{array}$ & $\begin{array}{c}S / N_{\text {noise }} \\
(\mathrm{dB})\end{array}$ & $\begin{array}{c}S / N_{\text {jamm }} \\
(\mathrm{dB})\end{array}$ \\
\hline 0.825 & 0.199 & 0 & - \\
0.85 & 0.209 & 0 & -30 \\
0.9 & 0.207 & 0 & -60 \\
0.375 & 0.171 & 6 & - \\
0.4 & 0.202 & 6 & -30 \\
0.475 & 0.226 & 6 & -60 \\
0.325 & 0.226 & 10 & - \\
0.35 & 0.167 & 10 & -30 \\
0.35 & 0.171 & 10 & -60 \\
0.2 & 0.176 & 20 & - \\
0.2 & 0.147 & 20 & -30 \\
0.2 & 0.180 & 20 & -60 \\
0.125 & 0.114 & 30 & - \\
0.15 & 0.096 & 30 & -30 \\
0.125 & 0.114 & 30 & -60 \\
\hline
\end{tabular}

subarray $X$ includes 23 "virtual" elements, at the outputs of which, samples $x_{k}^{(e)}(n)(1 \leq k \leq 23)$ are evaluated. Similarly, samples $x_{k}^{(e)}(n) 2 \leq k \leq 24$ are evaluated at the outputs of 23 "virtual" elements of subarray $Y$. Such preprocessing scheme allows to improve the TLS ESPRIT method applied for weaker signals, at $S / N_{\text {noise }}<6 \mathrm{~dB}$. The signal to noise ratio $S / N_{\text {noise }}$ has been determined at outputs of the matched filters MF, see Figure 4. The signal to interference (jamming) ratio $S / N_{\text {jamm }}$ has been determined at inputs of matched filters (MF). It is justified by fact, that jamming signals may be correlated to emitted chirp modulation pulse. 


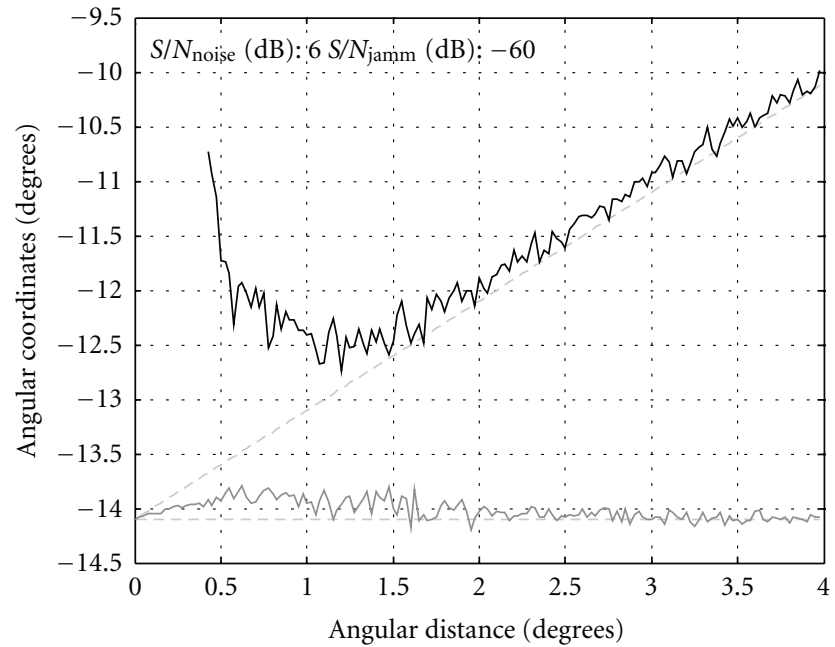

FIGURE 9: Histogram of polar coordinates evaluated by using the ESPRIT method at $S / N_{\text {noise }}=6 \mathrm{~dB}$ and $S / N_{\text {jamm }}=-60 \mathrm{~dB}$.

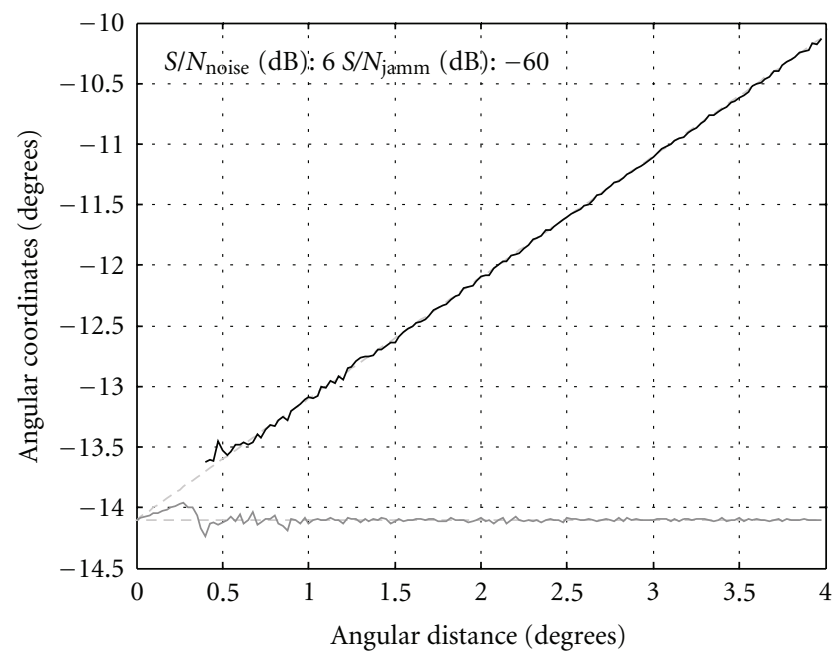

Figure 10: Histogram of polar coordinates evaluated by using the TLS ESPRIT method at $S / N_{\text {noise }}=6 \mathrm{~dB}$ and $S / N_{\text {jamm }}=-60 \mathrm{~dB}$.

\section{Estimation of Angular Coordinates in the Presence of Interferences}

An important advantage of the TLS ESPRIT method is its high immunity against interference. To illustrate this property, let us assume that a point-like interference source is located in the direction represented by angle $\theta_{\text {jamm }}=1.4^{\circ}$. It has been assumed also that the surface power density of the jamming signal is $60 \mathrm{~dB}$ higher than the surface power density of both useful signals. In the presence of such interference, conventional radar systems (with the receive patterns nonadapting to the interference) will be jammed. An effective way of suppressing the influence of interference is to use the adaptive antenna with digitally formed receive patterns. Such receive patterns should have deep minima (maximum attenuation) at directions from which the strong

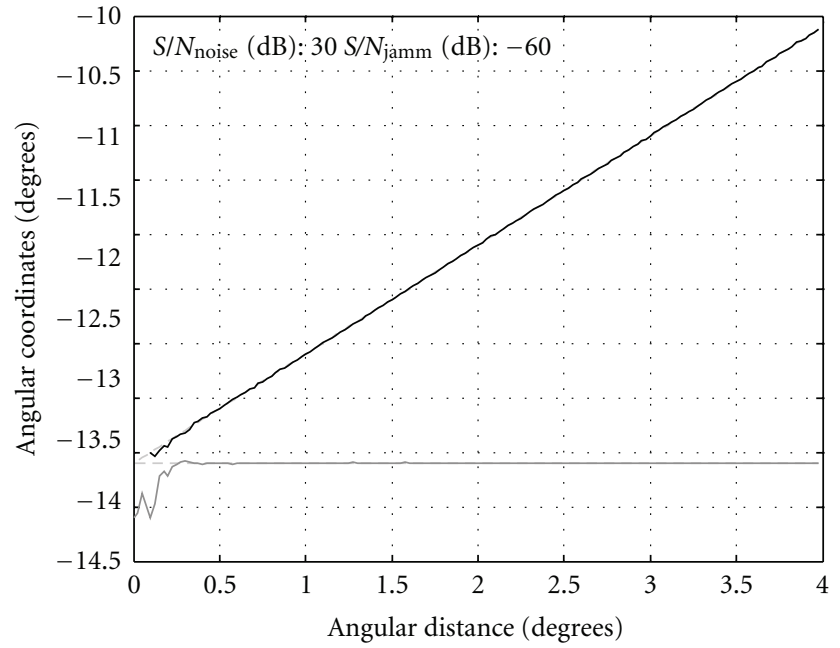

FIGURE 11: Histogram of polar coordinates evaluated by using the ESPRIT method at $S / N_{\text {noise }}=30 \mathrm{~dB}$ and $S / N_{\text {jamm }}=-60 \mathrm{~dB}$.

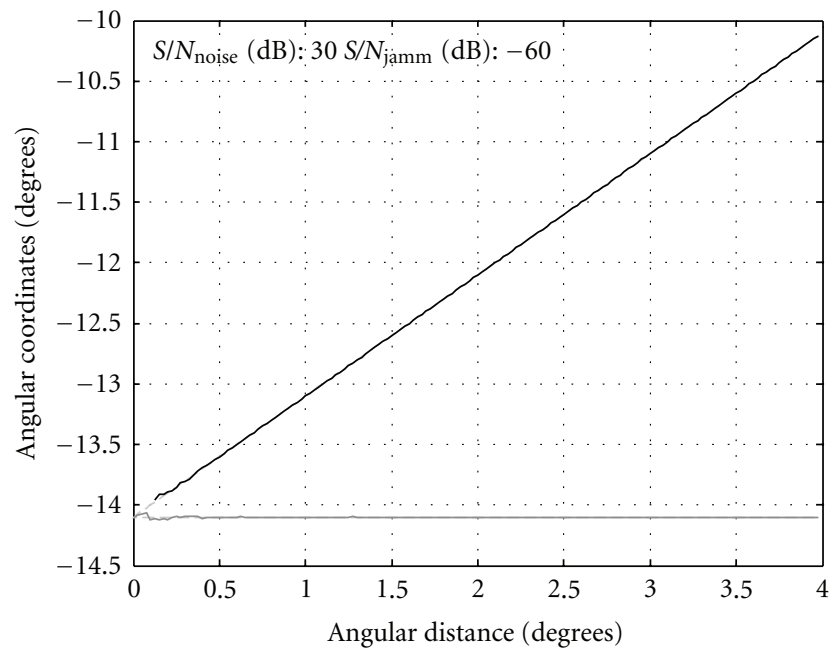

FIGURE 12: Histogram of polar coordinates evaluated by using the TLS ESPRIT method at $S / N_{\text {noise }}=30 \mathrm{~dB}$ and $S / N_{\text {jamm }}=-60 \mathrm{~dB}$.

interfering signals are received. The corresponding results of simulations are summarized in Table 1.

\section{Reducing the Errors of Angular Coordinates by Using Bursts with Variable Pulse Repetition Intervals}

The presence of the second object within the antenna beam causes errors of angular coordinates when both objects have similar or equal normalized Doppler frequencies. Fortunately, the TLS ESPRIT method under discussion is able to discriminate both objects and sources of interference when a variable pulse repetition interval is applied, see Figure 3. This property is confirmed by estimates of angles evaluated by using the TLS ESPRIT method. The corresponding results are illustrated in Figures 8, 10, and 12. All these results have 


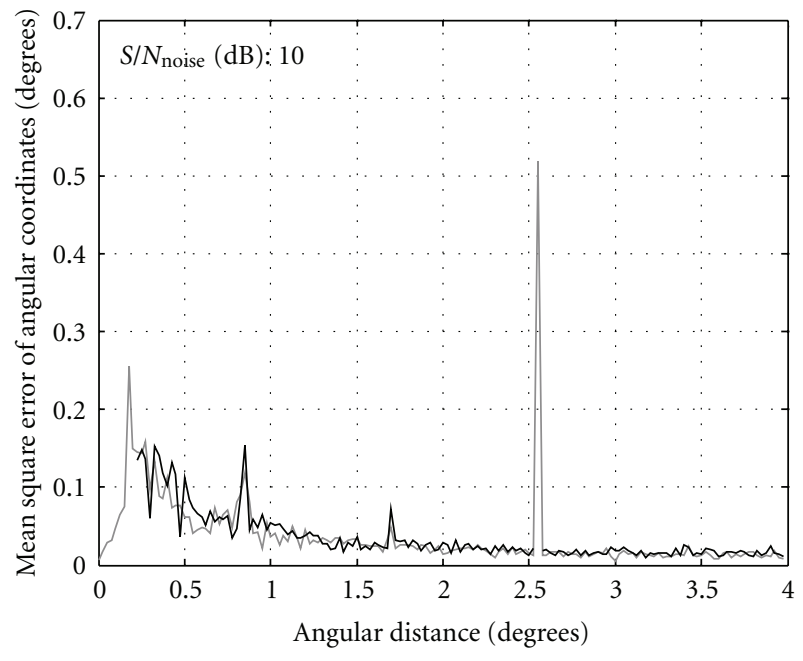

FIGURE 13: Histogram of mean square error of polar coordinates evaluated by using the TLS ESPRIT method at $S / N_{\text {noise }}=10 \mathrm{~dB}$, in absence of an external noise signal and a constant repetition time of radar pulses, $T_{p}=4 \mathrm{~ms}, v=1000 \mathrm{~m} / \mathrm{s}$.

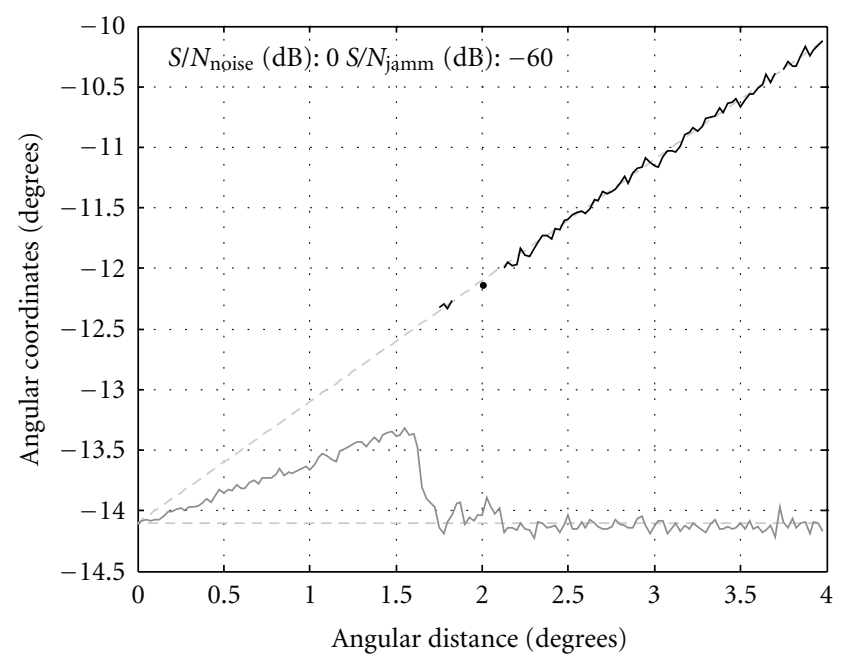

FIGURE 14: Histogram of polar coordinates evaluated by means of the TLS ESPRIT method at $S / N_{\text {noise }}=0 \mathrm{~dB}, S / N_{\text {jamm }}=-60 \mathrm{~dB}$ and without initial preprocessing.

been obtained by using the initial preprocessing described in Section 4. Simulations have been carried out for $S / N_{\text {noise }}=$ $0 \mathrm{~dB}, S / N_{\text {noise }}=6 \mathrm{~dB}$, and $S / N_{\text {noise }}=30 \mathrm{~dB}$, respectively. For comparison, the equivalent results, obtained by means of the ESPRIT method, are illustrated in Figures 7, 9, and 11, [7].

From the analysis of the diagrams obtained by means of the ESPRIT and the TLS ESPRIT method, it results that TLS ESPRIT offers better accuracy and higher resolution. The corresponding values of mean square errors for angular coordinates are given in Table 1.

Results presented in Figures 5-12 and Table 1 have been obtained for bursts with variable pulse repetition intervals. For comparison, Figure 13 presents mean square errors for angular coordinates occurring when bursts with the same

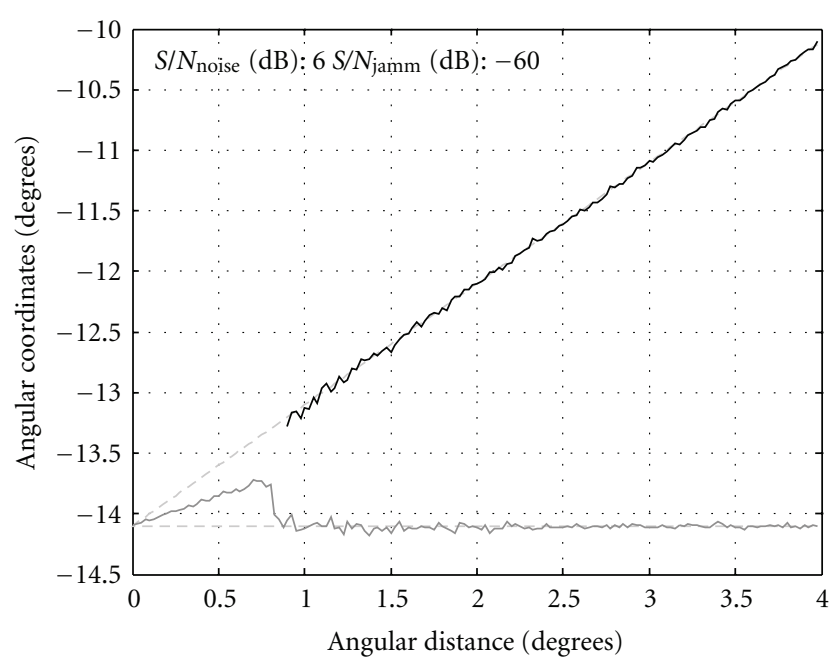

FIGURE 15: Histogram of polar coordinates evaluated by means of the TLS ESPRIT method at $S / N_{\text {noise }}=6 \mathrm{~dB}, S / N_{\text {jamm }}=-60 \mathrm{~dB}$, and without initial preprocessing.

pulse repetition interval are used and when the pulse repetition frequency is small ( $T_{p}=4 \mathrm{~ms}$ ) versus the Doppler frequency of signals reflected from objects moving with high velocity $(v=1000 \mathrm{~m} / \mathrm{s})$. The maximum of error observed in Figure 13 occurs when normalized Doppler frequencies of both objects are equal.

The comparison of results obtained by the TLS ESPRIT for signal samples from the 32-element antenna array to corresponding results obtained for signal samples formulated for equivalent "virtual" 24-element antenna array states that the use of preprocessing increases efficiency of the TLS ESPRIT for weaker signals, for which the $S / N_{\text {noise }}<6 \mathrm{~dB}$. The results presented in Figures 14 and 15 justify this conclusion.

From the analysis performed above, results that proposed additional preprocessing significantly improve the effectiveness of the TLS ESPRIT method in application under consideration.

\section{Conclusions}

The results of numerous computer simulations illustrated in Figures $6,8,10$, and 12 confirm the applicability of the TLS ESPRIT method to solve different variants of the problem under consideration. The comparison of these results with their correspondents obtained by using the ESPRIT method, see Figures 7, 9, and 11, indicates that the TLS ESPRIT method offers better angular resolution and more reliable estimation of angular coordinates of two moving objects, especially when weak echoes are being received in the presence of strong jamming signal. Another, also important, result of the performed simulations is the confirmation of the usefulness of the proposed signal preprocessing, the essence of which is expressed by formula (24). From the obtained results, see Figures 8, 10, 14, and 15, it follows that the proposed preprocessing contributes to increasing the accuracy of angular coordinates and consequently makes 
the TLS ESPRIT method more effective, naturally in the application under consideration. In other words, the preprocessing increases the effectiveness of the TLS ESPRIT method especially for the weaker echoes of useful signals. In the first approximation, this essential improvement can be explained in the following way: as a result of the addition of signals $x_{i}(n)$, see (24), the uncorrelated noise waveforms are summed. Thereby, in elements of antenna array, increasing of a signal-to-noise ratio is observed. In this paper, an emphasis has been put on the fact that the effective use of the TLS ESPRIT method requires an appropriate method of scanning. When scanning signals with identical pulse repetition intervals in all three bursts used, see Figure 3, significant errors may occur for some angle locations. This effect is illustrated by the simulation results shown in Figure 13. This parasitic effect can be reduced by changing pulse repetition interval in subsequent bursts, see Figure 3. Here, it deserves noting that bursts with variable pulse repetition intervals are widely used in order to avoid errors related to the so-called "blind speeds" $[2,9]$.

\section{References}

[1] A. I. Leonov and K. J. Fomichev, Monopulse Radars, Artech House, Norwood, Mass, USA, 1986.

[2] M. I. Skolnik, Introduction to Radar Systems, McGraw-Hill, New York, NY, USA, 2nd edition, 1980.

[3] B. Ottersten, M. Viberg, and T. Kailath, "Performance analysis of the total least squares ESPRIT algorithm," IEEE Transactions on Signal Processing, vol. 39, no. 5, pp. 1122-1135, 1991.

[4] A. Paulraj, R. Roy, and T. Kailath, "A subspace rotation approach to signal parameter estimation," Proceedings of the IEEE, vol. 74, no. 7, pp. 1044-1045, 1986.

[5] R. Roy, A. Paulraj, and T. Kailath, "ESPRIT—a subspace rotation approach to estimation of parameters of cisoids in noise," IEEE Transactions on Acoustics, Speech, and Signal Processing, vol. 34, no. 5, pp. 1340-1342, 1986.

[6] R. Roy and T. Kailath, "ESPRIT-estimation of signal parameters via rotational invariance techniques," IEEE Transactions on Acoustics, Speech, and Signal Processing, vol. 37, no. 7, pp. 984995, 1989.

[7] W. Rosłoniec, "Estimation of angular coordinates of two moving objects using the ESPRIT method," Elektronika, no. 1, pp. 119-128, 2010 (Polish).

[8] A. L. Swindlehurst, B. Ottersten, R. Roy, and T. Kailath, "Multiple invariance ESPRIT," IEEE Transactions on Signal Processing, vol. 40, no. 4, pp. 867-881, 1992.

[9] P. Z. Peebles Jr., Radar Principles, John Wiley \& Sons, New York, NY, USA, 1998. 

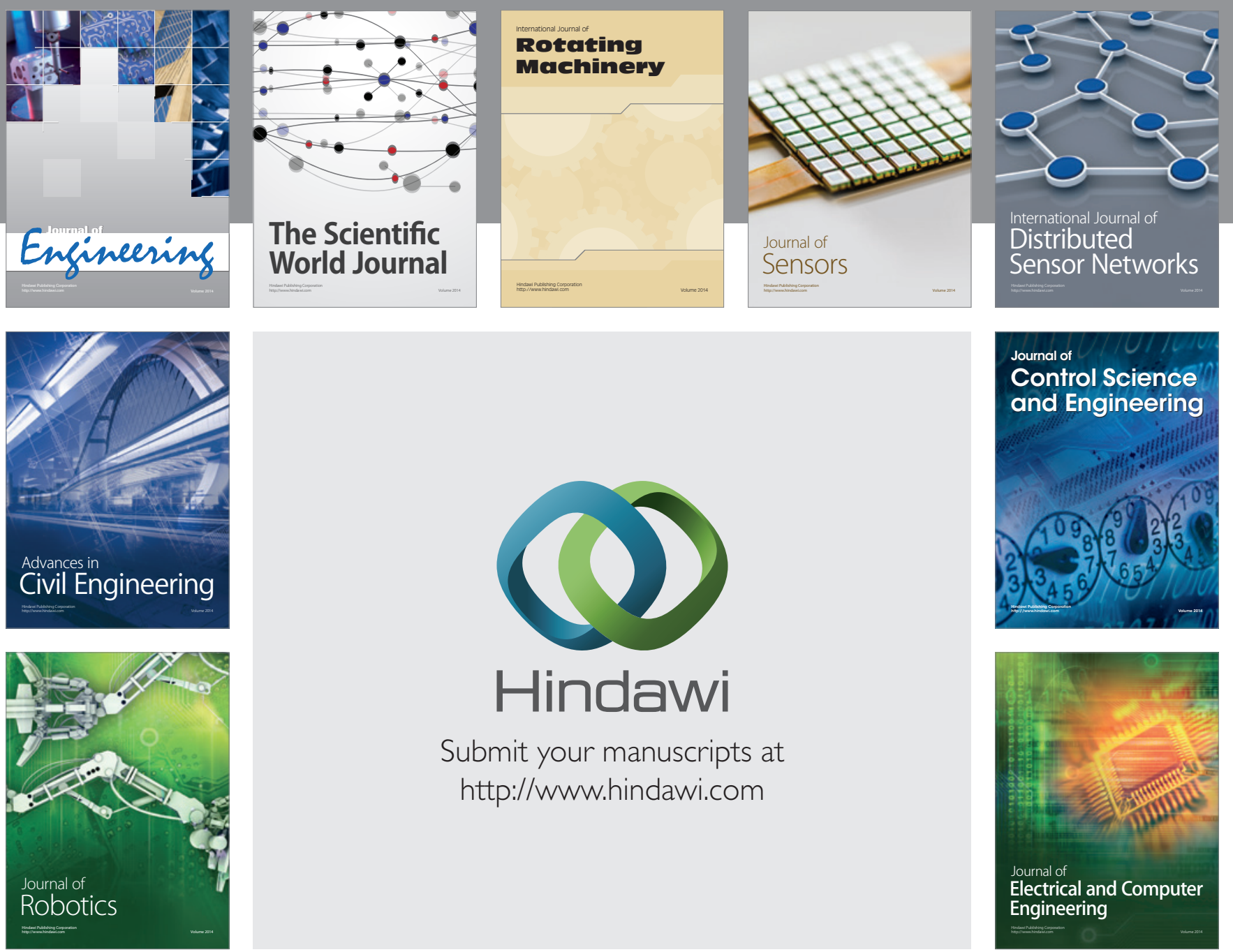

Submit your manuscripts at

http://www.hindawi.com
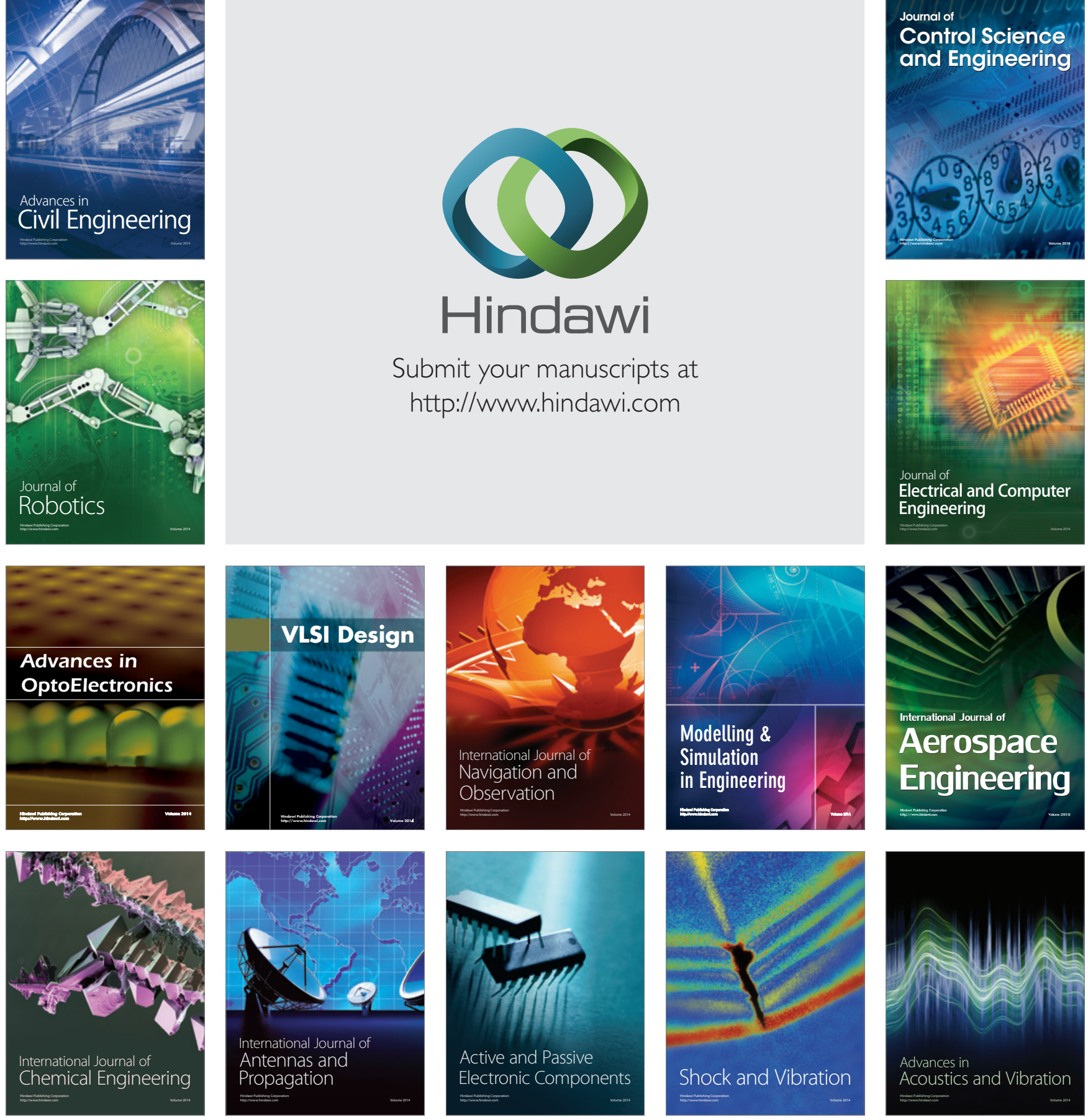